\title{
Total Knee Arthroplasty for Chronic Neglected Posterior Knee Dislocation: Case Report and Literature Review
}

\section{Amine Belmoubarik*, Merouane Abouchane, Mohammed Fahsi, Hamza Benameur, Mustapha Fadili, Mohammed Nechad}

Ibn Rushd University Hospital, Casablanca, Morocco

Email: ${ }^{*}$ aminovich2005@gmail.com, abouchane123@gmail.com, fahsimohammed@gmail.com, hamzabenameur@gmail.com, m.fadili@gmail.com, $\underline{\text { m.nechad@gmail.com }}$

Received 25 September 2015; accepted 13 October 2015; published 16 October 2015

Copyright (C) 2015 by authors and OALib.

This work is licensed under the Creative Commons Attribution International License (CC BY).

http://creativecommons.org/licenses/by/4.0/

(c) †) Open Access

\section{Abstract}

Knee dislocations are rare injuries. Posterior knee dislocations are only a small subset of them. There is a paucity of literature regarding the management of such neglected cases. We report here, a case of neglected irreducible posterior knee dislocation treated with a total knee arthroplasty followed by gradual rehabilitation with good outcome at 1 year follow-up.

\section{Keywords}

Knee Dislocation, Neglected, Total Knee Arthroplasty

Subject Areas: Orthopedics, Surgery \& Surgical Specialties

\section{Introduction}

Knee dislocation is a rare injury and results from high-energy trauma [1]. The purpose of the treatment of the acute dislocation is to obtain and maintain a reduced knee joint as well as addressing any concomitant neurovascular injuries [1]. Neglected knee dislocations and knee dislocations with delayed treatment are much more uncommon [2]. The usual treatment reported was conservative: arthrodesis [3] or ligament reconstruction [4]. However, only 3 cases of total knee arthroplasty (TKA) [1] [2] were described.

We report through discussion of the clinical and therapeutic approach, our experience in the management of this rarely encountered in daily practice dislocations are rare injuries. Posterolateral knee dislocations are only a small subset of them. There is a paucity of literature regarding the management of such neglected cases. We

${ }^{*}$ Corresponding author.

How to cite this paper: Belmoubarik, A., Abouchane, M., Fahsi, M., Benameur, H., Fadili, M. and Nechad, M. (2015) Total Knee Arthroplasty for Chronic Neglected Posterior Knee Dislocation: Case Report and Literature Review. Open Access Library Journal, 2: e1976. http://dx.doi.org/10.4236/oalib.1101976 
report here, a case of neglected irreducible posterolateral knee dislocation treated with open reduction and isolated posterior cruciate ligament reconstruction followed by gradual rehabilitation with good outcome at 3 years follow-up.

\section{Observation}

A 73-year-old man consults in our structure for persistent pain and deformation of the lower left leg at the knee for three months after a traffic accident. The patient presents a wound in his left knee that was sutured in the emergency without any radiological assessment; He couldn't walk or stand for 3 months. Physical examination revealed a deformed left knee in bayonet with antero-posterior enlargement (Figure 1) and a passive flexion of $30^{\circ}$ and $30^{\circ}$ to $150^{\circ}$ of motion without frontal laxity (Figure 2). The extensor mechanisms and the neurovascular functions were all intact. Radiographically, the left knee showed an unreduced posterior dislocation (Figure 3). After detailed explanations of the risks and benefits, a TKA of the left knee was performed. A midline incision using the conventional medial parapatellar approach to the knee was made. Intraoperative examination revealed a fixed posterior dislocation of the knee, and missing osteochondral surfaces over the lower part of the femoral trochlea and anterior tibia plateau and both anterior and posterior cruciate ligaments were absent (Figure 4) with contracture of the posterior soft tissues. Medial-lateral soft tissue balancing was performed first, followed by classical bony cuts at $6^{\circ}$ anatomical axis. A normal femoral component was chosen after a posterior soft-tissue release for balancing the flexion-extension gaps (the release was not important in the fear to touching the vascular axis). The patella was resurfaced, and posterior soft-tissue release was carried out extensively to allow full extension of the left knee with the components being tested in position. Flexion-extension and medial-lateral gaps were also checked to be nearly in balance, with relative stability during the full range of motion with the prostheses in place. Because stability was obtained using an LPS (Legated postero stabilized) type of implant. A constraining implant was not considered necessary. Finally, Nexgen Fixed-Flex total knee components (LPS type, Zimmer) were applied and fully cemented (Figure 5). The intraoperative range of motion was approximately $-10^{\circ}-160^{\circ}$ a posterior splint was applied to prevent a dislocation for 4 weeks. No dislocation has been noted and mobility was started in the fifth week without complication. At latest follow-up 1 year postoperatively, the man had a stable left knee with a range of motion of $-10^{\circ}-120^{\circ}$ and Lysholm à 80 . Radiographs showed a well aligned and well fixed total knee prosthesis (Figure 6). The patient was very satisfied with the final outcome.
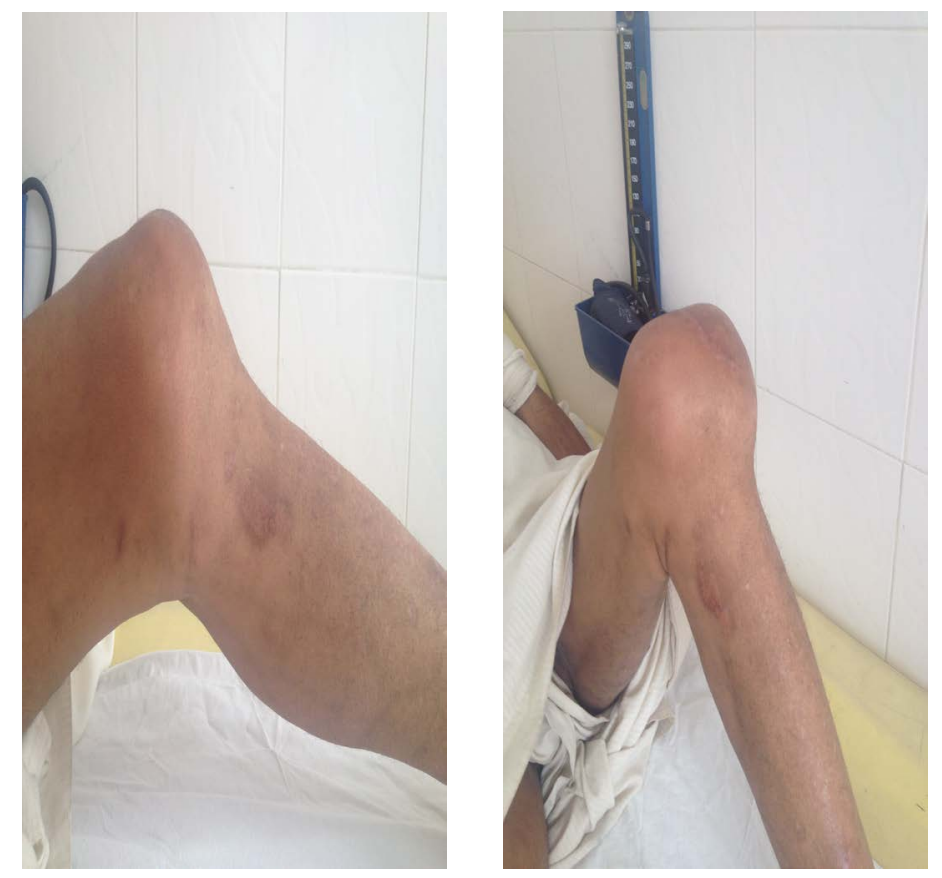

Figure 1. Deformed left knee in bayonet with antero-posterior enlargement. 

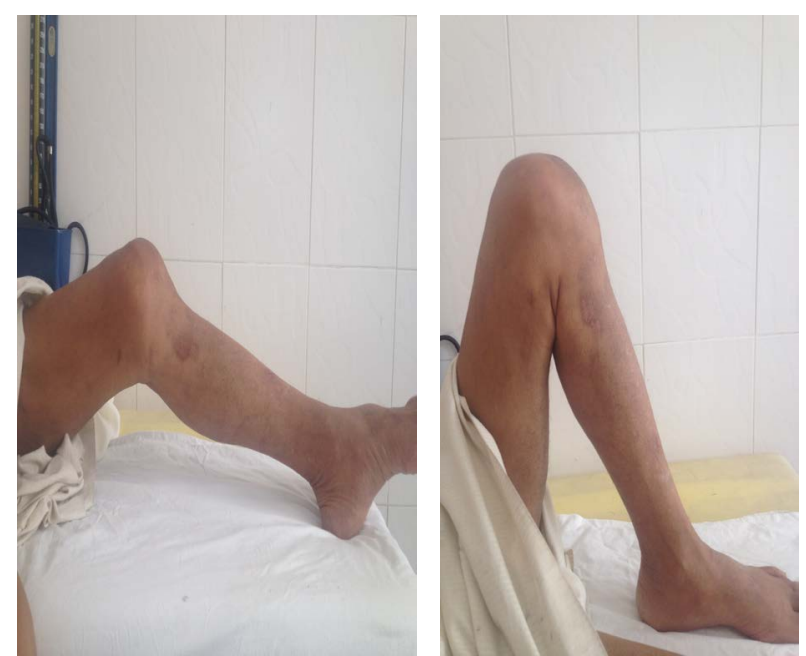

Figure 2. Passive flexion of $30^{\circ}$ and $30^{\circ}$ to $150^{\circ}$ of motion without frontal laxity.

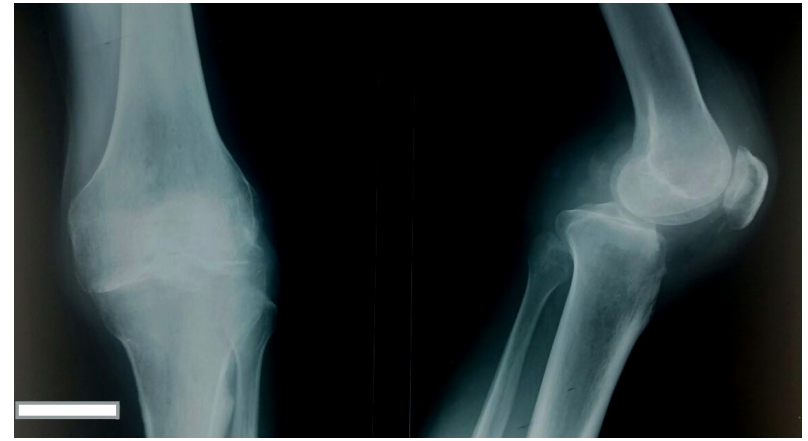

Figure 3. The left knee showed an unreduced posterior dislocation.

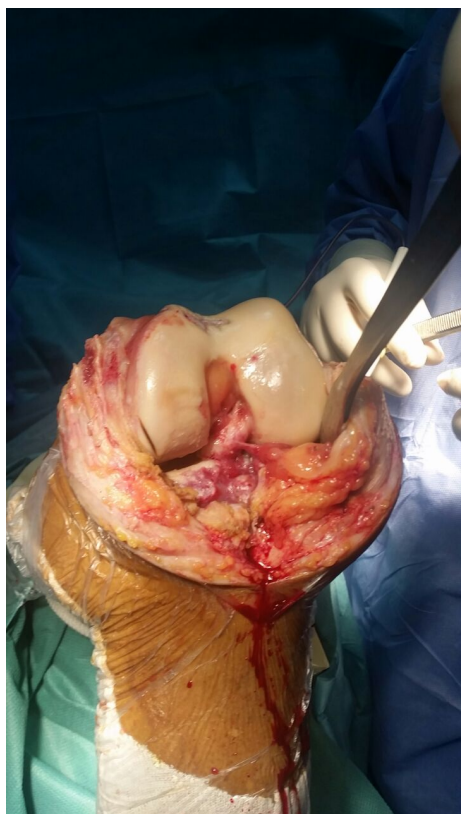

Figure 4. Intraoperative examination revealing a fixed posterior dislocation of the knee, and missing osteochondral surfaces over the lower part of the femoral trochlea and anterior tibia plateau and both anterior and posterior cruciate ligaments were absent. 


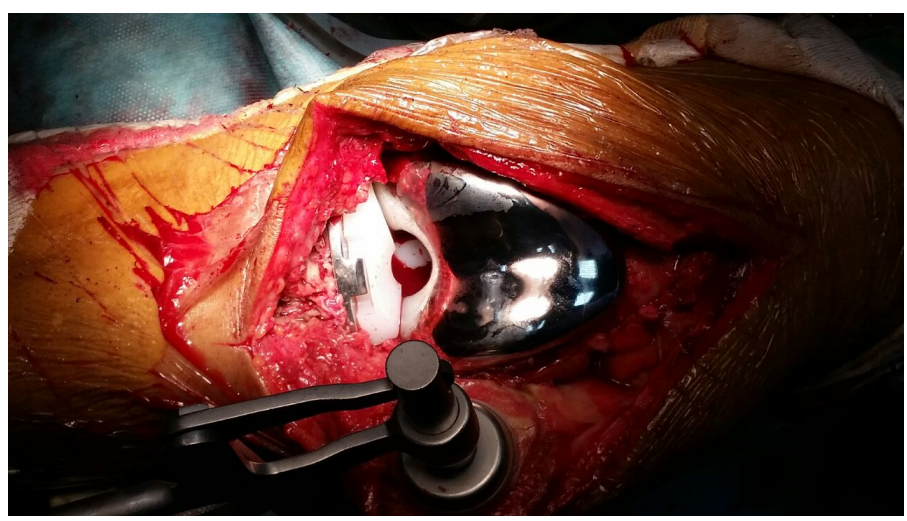

Figure 5. Nexgen Fixed-Flex total knee components (LPS type, Zimmer) fully cemented.

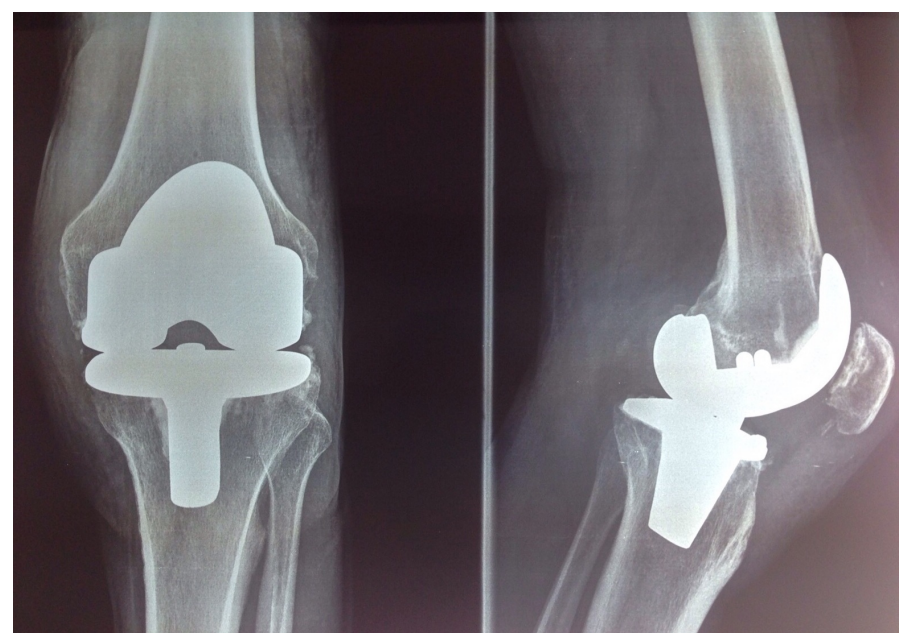

Figure 6. Radiographs showing a well aligned and well fixed total knee prosthesis.

\section{Discussion}

Chronic posterior knee dislocation and subluxation is a rare entity, presumably because most knee dislocations lend themselves to accurate diagnosis and appropriate treatment acutely [1]. There have been some case reports of delayed treatment of knee dislocation in the English literature [1] [3]-[7]. The time between the injury to the knee and definitive treatment ranged from 16 weeks to 8 months, and extensive open reconstruction using the Ilizarov technique, Steinmann pin fixation, ligament reconstruction or hinged external fixation have all been suggested [2]. For TKA, only 3 cases were reported in English literature [1] [2].

Petrie et al. reported two successful treatments with TKA and a constraining device for posterior knee dislocation 4 months after the initial injury. In their report, up-sizing the femoral component and posterior translation of the femoral components were recommended. In our case we didn't use a constraining implant with normal femoral component.

Chang reported the case of a TKA without constraining implants for a 56-year-old man with neglected knee dislocation injured 30 years previously, with several arthritis, the evolution was complicated by a posterior dislocation 5 days post operatively; Closed reduction was performed under spinal anesthesia, and a cylindrical cast was applied with the knee in $10^{\circ}$ of flexion. The cast was removed 4 weeks later, and physical therapy with range of motion exercises restarted without any subluxation or instability thereafter. So he recommended a constraining implant to avoid dislocation postoperatively. In our case also, we chose a appropriate femoral component with appropriate posterior translation of the femoral components with a posterior splint for 4 weeks which might have prevented further dislocation. 
In conclusion, we recommended TKA for neglected knee dislocation especially for elderly patients and/or with arthritis using constraining implants or posterior cast for 4 weeks to prevent a possible dislocation.

\section{Conclusion}

In conclusion, total knee arthroplasty is an effective and valuable treatment for chronic neglected dislocation of the knee and a constraining implant is recommended especially if the patient is aged or associated osteoarthritis.

\section{Conflicts of Interests}

No conflict of interest has been reported.

\section{Contribution of Authors}

All authors participated in the preparation of this manuscript.

\section{References}

[1] Petrie, R.S., Trousdale, R.T. and Cabanela, M.E. (2000) Total Knee Arthroplasty for Chronic Posterior Knee Dislocation: Report of Two Cases with Technical Considerations. Journal of Arthroplasty, 15, 380-386. http://dx.doi.org/10.1016/S0883-5403(00)90854-4

[2] Chen, H.-C. and Chiu, F.-Y. (2007) Chronic Knee Dislocation Treated with Arthroplasty. Injury Extra, 38, $258-261$. http://dx.doi.org/10.1016/j.injury.2006.10.025

[3] Henshaw, R.M., Shapiro, M.S. and Oppenheim, W.L. (1996) Delayed Reduction of Traumatic Knee Dislocation. A Case Report and Literature Review. Clinical Orthopaedics and Related Research, 330, 152-156. http://dx.doi.org/10.1097/00003086-199609000-00018

[4] Saini, R., Mootha, A.K., Goni, V.G. and Dhillon, M.S. (2010) Neglected Irreducible Posterolateral Knee Dislocation. Indian Journal of Orthopaedics, 44, 468-470. http://dx.doi.org/10.4103/0019-5413.69323

[5] Richter, M. and Lobenhoffer, P. (1998) Chronic Posterior Knee Dislocation: Treatment with Arthrolysis, Posterior Cruciate Ligament Reconstruction and Hinged External Fixation Device. Injury, 29, 546-549. http://dx.doi.org/10.1016/S0020-1383(98)00095-3

[6] Simonian, P.T., Wickiewicz, T.L., Hotchkiss, R.N., et al. (1998) Chronic Knee Dislocation: Reduction, Reconstruction, and Application of a Skeletally Fixed Knee Hinge. A Report of Two Cases. American Journal of Sports Medicine, 26, 591-596.

[7] Watanabe, K., Yamada, Y., Kura, H., et al. (2001) Chronic Knee Fracture Dislocation Treated by the Ilizarov Technique: Case Report. Journal of Trauma, 50, 151-154. http://dx.doi.org/10.1097/00005373-200101000-00033 